\title{
Arrhenius-law-governed homo- and heteroduplex dissociation
}

\author{
Matthias Bochtler $\mathbb{0}^{*}$ \\ IIMCB, Trojdena 4, 02-109 Warsaw, Poland \\ and Polish Academy of Sciences, IBB, Pawinskiego 5a, 02-106 Warsaw, Poland
}

(Received 7 January 2019; revised manuscript received 21 January 2020; accepted 14 February 2020;

published 9 March 2020)

\begin{abstract}
A simple model of temperature-increase-driven homo- or heteroduplex dissociation is analyzed. It features a temperature-independent association constant, and a dissociation constant that increases with temperature according to an Arrhenius law. The model is analytically tractable for quasiequilibrium conditions, for two special cases in the intermediate regime, and in the strongly irreversible regime. In the latter, the fraction of isolated components depends on temperature according to a Gumbel minimal value distribution. The model suggests a logarithmic dependence of the dissociation temperature on the rate of temperature increase. It further predicts that the dissociation occurs in a twice broader temperature interval for slow than fast temperature increase. Finally, the model points to a previously overlooked source of discrepancy between apparent van't Hoff and calorimetric enthalpies. Applied to short double stranded DNA, the model explains the dependence of the melting temperature on the rate of temperature increase, and the twice lower width of the melting transition in low salt compared to high salt conditions.
\end{abstract}

DOI: 10.1103/PhysRevE.101.032405

\section{INTRODUCTION}

\section{A. Thermodynamic model}

A simple model for temperature $T$ controlled homo$(A=B)$ or heteroduplex $(A \neq B)$ dissociation or fission, applicable, for example, to DNA melting, is considered. The model treats dissociation as a single-step event controlled by an Arrhenius law and assumes that dissociation is counteracted by a temperature-independent association reaction. Let $T$ denote the temperature, and $T_{m}$ the temperature for which $50 \%$ of the homo- or heteroduplex is dissociated (" $m$ " stands for "median") under quasiequilibrium conditions. Further, use $k_{\text {dis }}(T)$ for the temperature-dependent dissociation rate constant, $k$ for the dissociation rate constant at temperature $T_{m}$, and $k_{\text {as }}$ for the association rate constant. $R$ is the Boltzmann constant, and $\Delta H$ the dissociation enthalpy. Then the above model can be summarized as follows:

$$
\begin{gathered}
A B \stackrel{k_{\mathrm{dis}}(T)=k e^{\Theta(T)}}{\longrightarrow} A+B, \\
A+B \stackrel{k_{\mathrm{as}}(T)=\mathrm{const}}{\longrightarrow} A B, \\
\Theta(T)=\Delta H / R \cdot\left(1 / T_{m}-1 / T\right) .
\end{gathered}
$$

The larger the dissociation enthalpy $\Delta H$, the more abruptly dissociation occurs when the temperature is increased. Quantitatively, this is expressed by the van't Hoff width $\Delta T_{\mathrm{vH}}$,

\footnotetext{
*mbochtler@iimcb.gov.pl

Published by the American Physical Society under the terms of the Creative Commons Attribution 4.0 International license. Further distribution of this work must maintain attribution to the author(s) and the published article's title, journal citation, and DOI.
}

which describes the temperature range of dissociation (in quasiequilibrium conditions):

$$
\frac{\Delta T_{\mathrm{vH}}}{4}=R T_{m}^{2} / \Delta H
$$

The abruptness of the dissociation reaction depends on the dimensionless parameter $\xi$, defined as

$$
\xi=\frac{\Delta T_{\mathrm{vH}} / 4}{T_{m}}=\frac{R T_{m}}{\Delta H}
$$

In the following, we assume that the transition is well defined, i.e., $\xi \ll 1$. Temperature is expressed by the dimensionless parameter $\theta$, which measures the difference to $T_{m}$, in units of $\Delta T_{\mathrm{vH}} / 4$ :

$$
\theta=\frac{T-T_{m}}{\frac{\Delta T_{\mathrm{vH}}}{4}}
$$

In terms of the dimensionless $\xi$ and $\theta$, the Boltzmann exponent of Eq. (3) takes the form

$$
\Theta(\theta, \xi)=\frac{1}{\xi}\left(1-\frac{1}{1+\xi \theta}\right) \approx \theta\left(1-\xi \theta+(\xi \theta)^{2}-\cdots\right) .
$$

Note that $\theta$ can only vary between $-1 / \xi$ and $\infty$, because temperature has to be positive. The corresponding range for $\Theta$ is from $-\infty$ to $1 / \xi$. The expansion in Eq. (7) is valid for $|\xi \theta| \ll 1$.

\section{B. Kinetic implications of the model}

Consider the kinetic consequences of the model for homoduplex dissociation first: Let the reaction begin with only $A_{2}$. Let $c(A)$ and $c\left(A_{2}\right)$ denote actual concentrations of $A$ and $A_{2}$, respectively. Also let $c_{0}$ denote the concentration of homoduplexes at the beginning, so that $c(A) / 2+c\left(A_{2}\right)=c_{0}$ 
at any time $t$. Define $y$ as the fraction of unbound $A$, i.e., $y=$ $c(A) /\left[c(A)+2 c\left(A_{2}\right)\right]=c(A) /\left(2 c_{0}\right)$. Then $c(A)=2 y c_{0}$ and $c\left(A_{2}\right)=(1-y) c_{0}$. Formation of new $A_{2}$ reduces $c(A)$ at a rate $-d c(A) / d t=2 k_{\mathrm{as}} c^{2}(A)=8 k_{\mathrm{as}} y^{2} c_{0}^{2}$. Dissociation of $A_{2}$ adds to $c(A)$ at a rate $d c(A) / d t=2 k_{\mathrm{dis}} c\left(A_{2}\right)=2 k(1-y) e^{\Theta} c_{0}$. At $T_{m}$, the two rates should be equal. At temperature $T_{m}, y=1 / 2$, $\Theta=0, k_{\mathrm{dis}}=k$, and hence $2 k_{\mathrm{as}} c_{0}=k$. In the general case, the rates may not be balanced, and a change of $c(A)$ and hence $y$ may occur in time $t$. In the differential equation, the linear and quadratic terms in $y$ account for the dissociation and association rates, respectively:

$$
\frac{d y}{d(k t)}=-2 y^{2}+(1-y) e^{\Theta} .
$$

The argument for temperature-increase-driven heteroduplex dissociation is similar, but the prefactors are different. Define $c_{0}$ as the initial concentration of $A B$, and $y$ as the unbound fraction $y=c(A) /[c(A)+c(A B)]=c(B) /[c(A)+$ $c(A B)]$ of $A$ or $B$ at any time $t$. Then $c(A)=c(B)=y c_{0}$ and $c(A B)=(1-y) c_{0}$. Formation of new $A B$ reduces $c(A)$ at a rate $-d c(A) / d t=k_{\text {as }} c(A) c(B)=k_{\text {as }} y^{2} c_{0}^{2}$. Dissociation of $A B$ adds to $c(A)$ at a rate $d c(A) / d t=k_{\mathrm{dis}} c(A B)=k(1-y) e^{\Theta} c_{0}$. Analysis at $T_{m}, y=1 / 2$ leads to $k_{\mathrm{as}} c_{0} / 2=k$. At other temperatures, changes in $c(A)$ and $y$ can occur. Surprisingly, these are described by Eq. (8), originally derived for homoduplexes. A common equation is possible because differences between the homo- and heteroduplex cases are absorbed into the definition of the reference temperature.

\section{THEORETICAL RESULTS}

\section{A. Master equation for temperature-increase-driven dissociation}

Here, we consider temperature-increase-driven dissociation. It is assumed that temperature increases at a fixed rate $\Gamma$. Natural units for time and temperature are set by the inverse of the dissociation constant $k=k_{\mathrm{dis}}\left(T_{m}\right)$ and by $\Delta H_{\mathrm{vH}} / 4$. With reference to these units, the rate of temperature increase $\Gamma$ can be expressed as a dimensionless parameter $\alpha$ :

$$
\alpha=\frac{\Gamma}{k \frac{\Delta T_{\mathrm{vH}}}{4}} .
$$

Using Eq. (7), and eliminating time from Eq. (8), a master equation for the dissociation process can be derived that depends on parameters $\xi$ and $\alpha$ :

$$
d y / d \theta=\left[-2 y^{2}+(1-y) e^{\Theta(\theta, \xi)}\right] / \alpha, \quad y \stackrel{\theta \rightarrow-1 / \xi}{\longrightarrow} 0 .
$$

The parameter $\xi$, defined in Eq. (5), is a material property. The more abrupt the dissociation reaction (under equilibrium conditions), the smaller $\xi$. The parameter $\alpha$, defined in Eq. (9), depends on a combination of material properties and the rate of temperature increase $\Gamma$. According to Eq. (9), small and large values of $\alpha$ represent slow and fast temperature increases, respectively. As $\alpha$ quantifies rates of temperature increase with reference to units of time and temperature set by material properties, it is also possible to vary $\alpha$ for fixed heating rate $\Gamma$ by altering the dissociation constant $k$, a material property.
Formally, $\theta$ runs from $-1 / \xi$ to $\infty$, and $\Theta$ from $-\infty$ to $1 / \xi$. In practice, the dissociation reaction occurs in a fairly narrow $\theta$ interval (later in this work shown to have a FWHM of 5.1 or less). Dissociation is complete, i.e., $y \rightarrow 1$ for $\theta \rightarrow 0$ in the $\xi \rightarrow 0$ limit. For nonzero $\xi$, a further $y$ increase becomes impossible when $-2 y^{2}+(1-y) e^{1 / \xi}=0$. Therefore, the maximal value for $y$ is

$$
y_{\max }=\frac{e^{1 / \xi}}{4}\left(\sqrt{1+8 e^{-1 / \xi}}-1\right) \approx 1-2 e^{-1 / \xi} .
$$

$y_{\max }$ has a value close to, but not exactly equal to 1 , for $0<\xi \ll 1$. It is therefore convenient to define a normalized variable:

$$
\tilde{y}(\theta)=\frac{y(\theta)}{y_{\max }} .
$$

\section{B. "Quasistatistical" descriptors of the dissociation curve}

For all $\xi$, the variable $\tilde{y}(\theta)$ starts out at 0 for very low temperature $\theta$, grows with temperature, and eventually reaches a value of 1 in the high-temperature limit. Therefore, $\tilde{y}(\theta)$ and $d \tilde{y} / d \theta$ can be formally considered as a "cumulative probability" and "probability density" for the "random" variable $\hat{\theta}$. With respect to this variable, the dissociation curve peak position can be described by the average $\theta_{\mathrm{av}}$ or median $\theta_{\text {med }}$, the width by the standard deviation $\Delta \theta$, and the shape by the skewness $s$. The peak position indicator $\theta_{\text {med }}$ is calculated directly from $\tilde{y}\left(\theta_{\text {med }}\right)=1 / 2$, the other three quantities can be derived directly from the statistical moments:

$$
\begin{gathered}
\theta_{\mathrm{av}}=\langle\hat{\theta}\rangle, \\
\Delta \theta=\sqrt{\left\langle\hat{\theta}^{2}\right\rangle-\langle\hat{\theta}\rangle^{2}}, \\
s=\frac{\left\langle\hat{\theta}^{3}\right\rangle-3(\Delta \theta)^{2}\langle\hat{\theta}\rangle-\langle\hat{\theta}\rangle^{3}}{(\Delta \theta)^{3 / 2}} .
\end{gathered}
$$

In some situations, these "quasistatistical" measures are easier to calculate analytically than the more usual $\theta_{\text {peak }}$ and $\Delta \theta_{1 / 2}$ for peak position and half-width at half-maximum, respectively.

\section{Apparent dissociation constant and van't Hoff enthalpy}

For any fixed $\alpha$ and $\xi$, an apparent dissociation constant $K_{\text {app }}$ can be defined by analogy with its definition under equilibrium conditions. When $c_{0}$ denotes the total concentration of homo- or heteroduplex at the outset of the dissociation reaction:

$$
K_{\text {app }}(\theta)= \begin{cases}\frac{c^{2}(A)}{c\left(A_{2}\right)}=4 \frac{y^{2}}{1-y} c_{0} & \text { for homoduplexes } \\ \frac{c(A) c(B)}{c(A B)}=\frac{y^{2}}{1-y} c_{0} & \text { for heteroduplexes. }\end{cases}
$$

The van't Hoff enthalpy is related to the equilibrium constant $K_{\text {eq }}$ by the rule $\Delta H=-R d\left(\ln K_{\text {eq }}\right) / d(1 / T)$. The same expression can be used to define an apparent van't Hoff enthalpy $\Delta H_{\text {app }}$ in the nonequilibrium case:

$$
\Delta H_{\mathrm{app}}=-R \cdot \frac{d\left(\ln K_{\mathrm{app}}\right)}{d(1 / T)}=-R T^{2} \frac{d\left(\ln K_{\mathrm{app}}\right)}{d T} .
$$


Combining Eqs. (5), (9), and (17), one can then show that the ratio $\kappa$ of apparent and "true" van't Hoff enthalpy is

$$
\kappa=\frac{\Delta H_{\mathrm{app}}}{\Delta H}=(1+\xi \theta)^{2} \frac{d\left(\ln K_{\mathrm{app}}\right)}{d \theta}=\frac{d\left(\ln K_{\mathrm{app}}\right)}{d \Theta} .
$$

To measure $\kappa$ or $K_{\text {app }}$ accurately, both $y$ and $1-y$ need to be well defined, so neither can be close to zero. Determinations of $K_{\text {app }}$ therefore have to be made near $y=1 / 2$. To simplify the analysis, we assume here that they are made in the immediate vicinity. Notice that by definition of $K_{\text {app }}, d\left(\ln K_{\text {app }}\right)=$ $[2 / y+1 /(1-y)] d y$. The expression for $d\left(\ln K_{\text {app }}\right) / d \theta$ can then be simplified to $6 d y /\left.d \theta\right|_{y=1 / 2}$. Therefore, the ratio of apparent and true van't Hoff enthalpy for this typical case becomes according to Eq. (18):

$$
\kappa_{y=1 / 2}=\left.6\left(1+\xi \theta_{\mathrm{med}}\right)^{2} \frac{d y}{d \theta}\right|_{y=\frac{1}{2}}=\left.6 \frac{d y}{d \Theta}\right|_{y=1 / 2} .
$$

\section{Entropy change and dissipated entropy}

For a fixed $\xi$, let $\theta_{\alpha}(y)$ denote the dependence of $\theta$ on $y$ for a given $\alpha[y(\theta)$ is monotonically increasing and can therefore be inverted]. The entropy extracted from the environment into the system can be expressed in terms of integrals running from 0 to $y_{\max }$, as defined in Eq. (11):

$$
\begin{aligned}
\frac{\Delta S_{\alpha}}{R} & =\int \frac{\Delta H}{T_{m}+\theta_{\alpha} \frac{\Delta T_{\mathrm{vH}}}{4}} d y \\
& =\frac{1}{\xi} \int \frac{1}{1+\xi \theta_{\alpha}} d y=\frac{1}{\xi}-\int \Theta\left(\theta_{\alpha}, \xi\right) d y .
\end{aligned}
$$

In quasistationary conditions, the extracted entropy can be calculated analytically. As $\Theta=\ln \left[2 y^{2} /(1-y)\right]$ and $y_{\max }=1$ according to Eqs. (8) and (11), the identity $\int_{0}^{1} \ln x d x=-1$ can be used to derive

$$
\frac{\Delta S_{0}}{R}=\frac{1}{\xi}+1-\ln 2 .
$$

In nonequilibrium conditions, new entropy $S_{\text {dis }}$ is created, because less entropy is extracted from the reservoir than under equilibrium conditions. According to Eqs. (20) and (21),

$$
\frac{S_{\mathrm{dis}}}{R}=\frac{\Delta S_{0}-\Delta S_{\alpha}}{R}=1-\ln 2+\int_{0}^{y_{\max }} \Theta\left(\theta_{\alpha}, \xi\right) d y .
$$

\section{THE $\xi \rightarrow 0$ LIMIT}

\section{A. One parameter master equation}

The dissociation kinetics become analytically tractable for extremely sharp transitions in the $\xi \rightarrow 0$ limit. In this limit, $|\xi \theta| \ll 1$ applies, and therefore Eqs. (7) and (8) simplify to

$$
\begin{gathered}
\Theta=\theta \text { for } \xi \rightarrow 0, \\
d y / d \theta=\left[-2 y^{2}+(1-y) e^{\theta}\right] / \alpha \quad y \stackrel{\theta \rightarrow-\infty}{\longrightarrow} 0 .
\end{gathered}
$$

Technically, the limit $\theta \rightarrow-\infty$ is incompatible with the condition $|\xi \theta| \ll 1$ required for the applicability of Eq. (23). However, master Eq. (24) predicts $y$ and $d y / d \theta$ close to 0 for large negative $\theta$. Therefore, the limit can formally be extended to $-\infty$ to simplify calculations. By an analogous argument, extension to $\theta \rightarrow \infty$ can also be justified. Note that in the $\xi \rightarrow 0$ limit, the expressions for $\tilde{y}$ of Eq. (12), the van't Hoff correction factor $\kappa$ of Eq. (18), and the dissipated entropy $S_{\text {dis }}$ of Eq. (22) can be simplified:

$$
\begin{gathered}
\tilde{y}=y, \\
\kappa=d\left(\ln K_{\text {app }}\right) / d \theta, \\
S_{\text {dis }} / R=\theta_{\mathrm{av}}+1-\ln 2 .
\end{gathered}
$$

\section{B. Numerical results for the dissociation curve}

The master Eq. (24) was solved for selected values $\alpha$, initially numerically (Fig. 1). The range of the $\alpha$ values was then explored in much greater detail, and characteristic parameters of the $y(\theta)$ and $d y / d \theta$ curves were determined as a function of $\alpha$ (Fig. 2). Several trends are apparent from the numerical solution: (a) The smaller $\alpha \geqslant 0$, the more the reaction approaches the quasiequilibrium case. (b) The larger $\alpha$, the higher the apparent dissociation temperature. (c) For large $\alpha \gg 1$, this dependence is logarithmic, for all measures of dissociation temperature. (d) For large $\alpha$, the shape of the dissociation curve becomes universal. (e) The dissociation transition becomes sharper as the rate of temperature increase goes up. The width of the transition (in temperature) decreases about two-fold, for either the half-width or the standard deviation criterion. (f) The dissociation curve is left-skewed for most of the $\alpha$ range. The skewness $s$ starts out from the low-temperature limiting value $s \approx-0.79$ to reach a value of $s=0$ for the first time at $\alpha \approx 1.002$. It then rises further to its maximal value $s \approx 0.014$, for $\alpha \approx 1.414$. Above this $\alpha$, skewness decreases again, reaches $s=0$ a second time for $\alpha \approx 1.998$, and then decreases further to the high-temperature limiting value of $s \approx-1.14$. Note that $d y / \theta$ is more leftskewed in the high-temperature than in the low-temperature limit.

\section{Numerical results for apparent van't Hoff enthalpy}

The dependence of $K_{\text {app }}$ on $\theta$ was determined using the previously determined data for $y(\theta)$ in combination with Eq. (16). In quasiequilibrium conditions, the dependence of $\ln K_{\text {app }}$ on temperature is given by the standard straight line in the van't Hoff plot. In the units used in this work, the slope of this line is 1 . For nonequilibrium melting, the line becomes kinked. In the high-temperature region, it continues to coincide with the quasiequilibrium result, but in the low-temperature region, it is twice steeper [Fig. 3(a)]. Numerical calculations indicate that for extremely large $\alpha$, the slope of the $\ln K_{\text {app }}$ versus $\theta$ plot can even exceed 2 (not shown, because of uncertainty regarding numerical stability of the results). As seen best from the $d\left(\ln K_{\text {app }}\right) / d \theta$ plot, the temperature at which the transition occurs is higher for large than small $\alpha$ [Fig. 3(b)]. Extrapolating to $\alpha \rightarrow 0$, one can expect that eventually the entire temperature range belongs to the high-temperature regime in which $d\left(\ln K_{\mathrm{app}}\right) / d \theta=1$. The numerically derived results are consistent with analytical results in special cases.

\section{Analytic solution for $\alpha>0$, low- and high-temperature limits}

To solve master Eq. (24) in the low- and high-temperature limits, it is useful to replace the independent variable $\theta$ by 

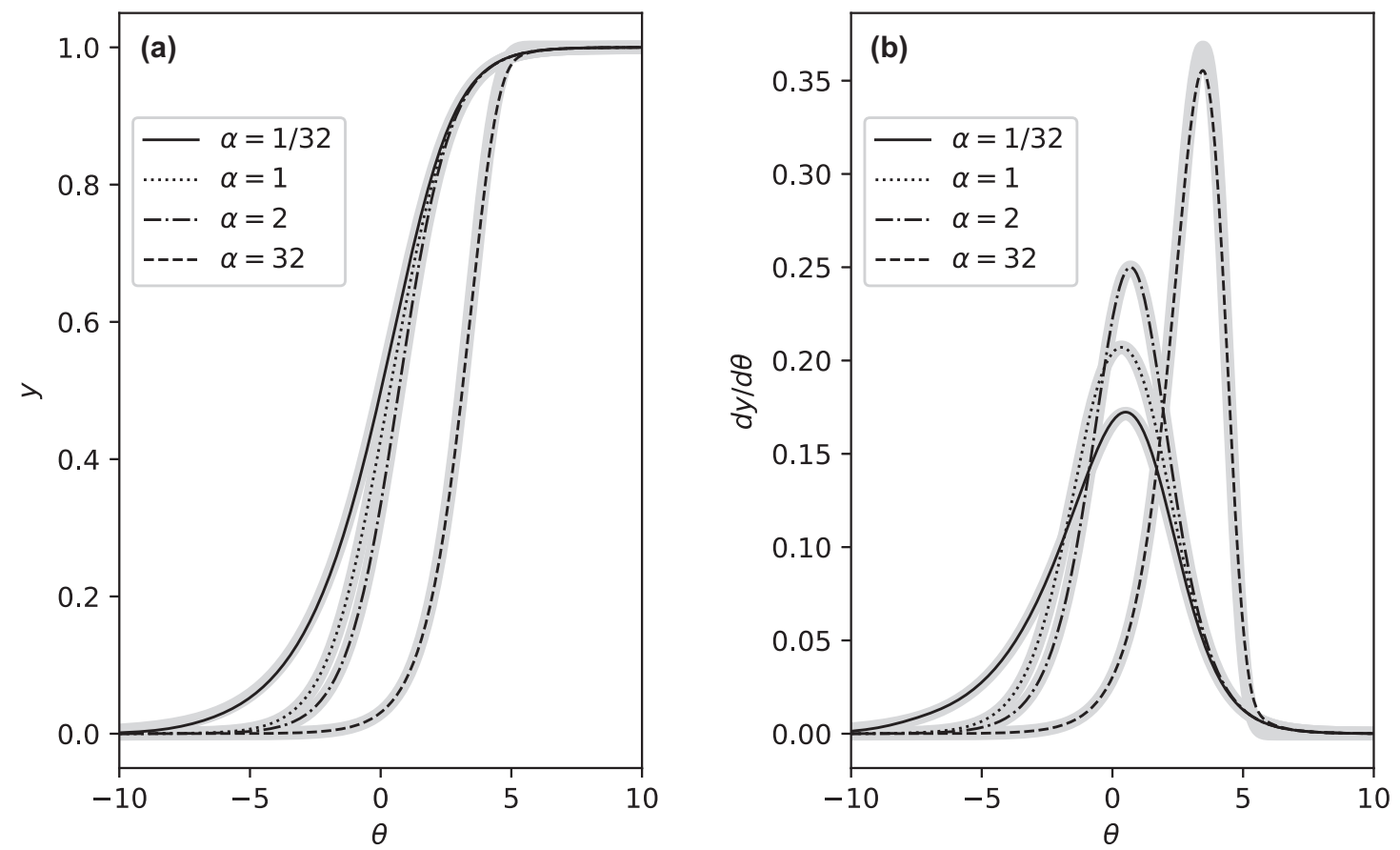

FIG. 1. Predicted dependence of the free fraction $y$ and its derivative $d y / d \theta$ on $\theta$ in the $\xi \rightarrow 0$ limit. Black curves are based on numerical integration of master Eq. (24). The gray curves below show the analytical solutions for quasiequilibrium [Eq. (32)], $\alpha=1$ [Eq. (42)], $\alpha=2$ [Eq. (76)], and large $\alpha$ [Eq. (61)].
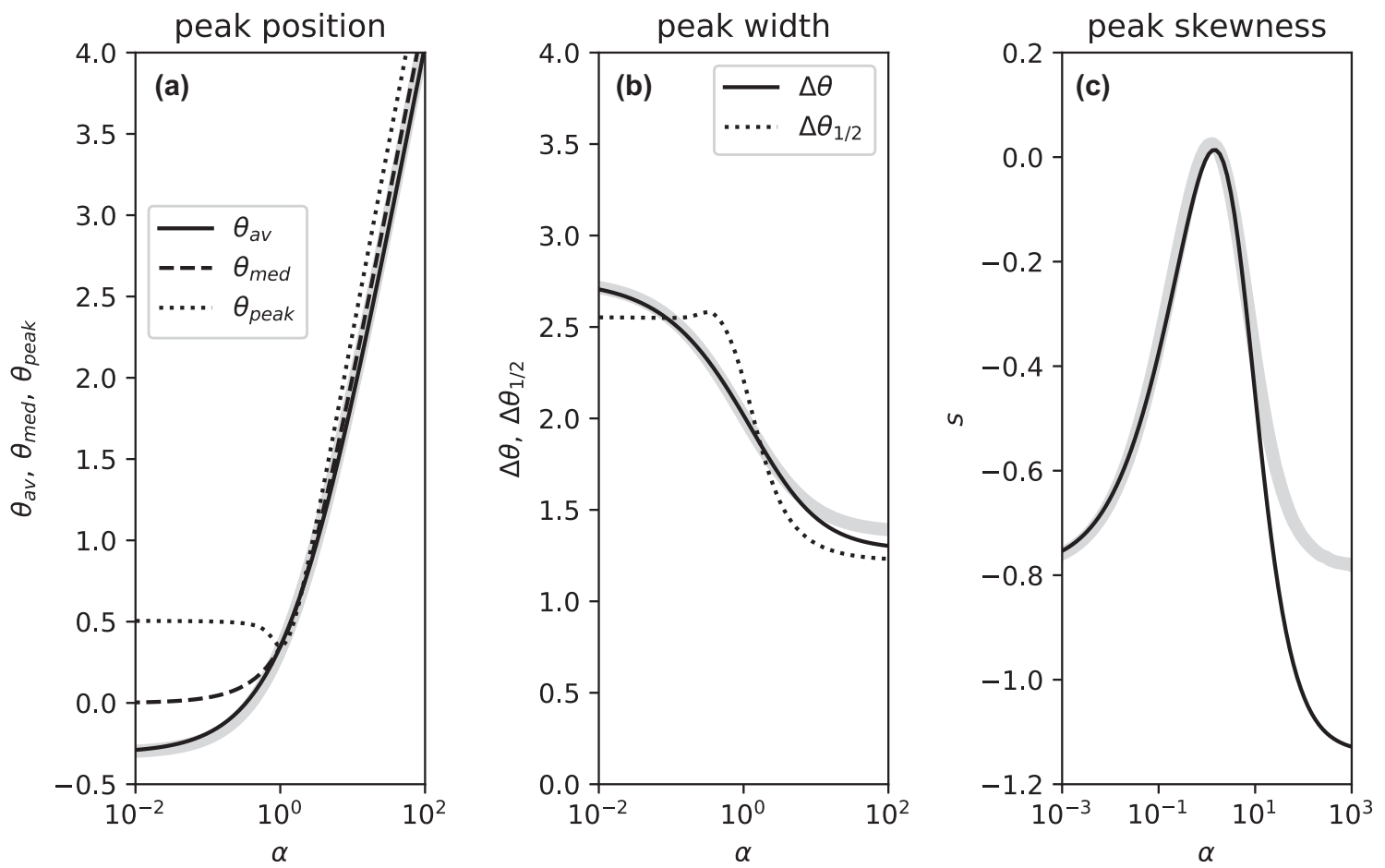

FIG. 2. Characteristics of the dissociation curves $y(\theta)$ for various $\alpha$ in the $\xi \rightarrow 0$ limit. The black curves are based on numerical solution of the master Eq. (24). The gray curves for $\theta_{\mathrm{av}}, \Delta \theta$ and $s$ are based on the approximate solution in Eqs. (71) and (72), for $f(\alpha)=\alpha^{2} / 2$. Note that the approximate solution predicts an incorrect asymptotic $s$ value for large $\alpha$. The correct value is obtained from the Gumbel solution of Eq. (61), according to Eq. (66) (see Table I). 

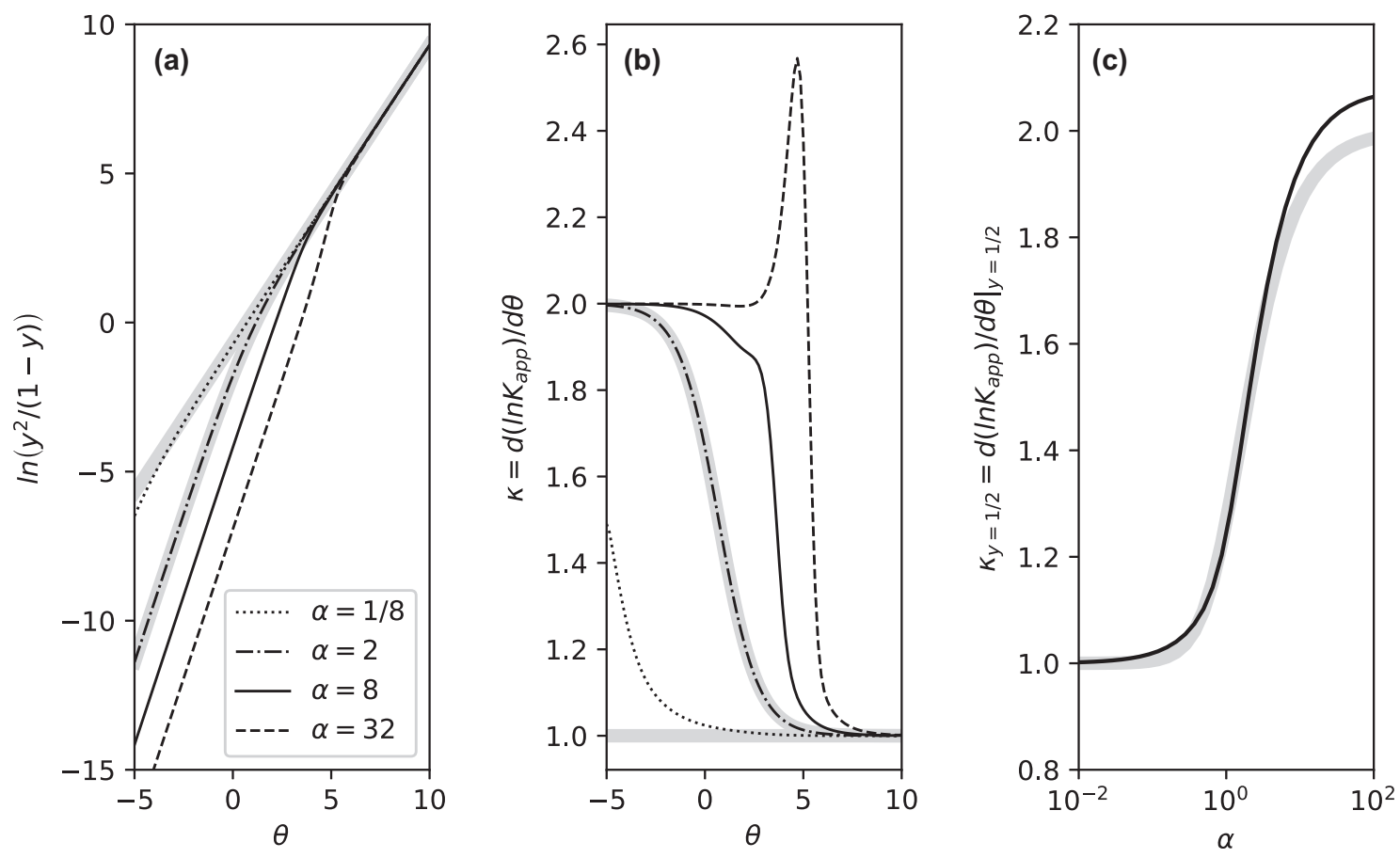

FIG. 3. Van't Hoff analysis in the $\xi \rightarrow 0$ limit. Panels (a) and (b) $\ln \frac{y^{2}}{1-y}$ and $d\left(\ln K_{\text {app }}\right) / d \theta$ as a function of temperature $\theta$, for selected parameters $\alpha$. Black lines show results for the numerical solution of the master equation, faint gray lines are analytical results in the quasiequilibrium limit, and for the special case $\alpha=2$. The legend in panel (a) applies also to panel (b). In the $\xi \rightarrow 0$ limit, the ratio $\kappa=\Delta H_{\text {app }} / \Delta H$ is equal to $d\left(\ln K_{\text {app }}\right) / d \theta$. Measurements of $K_{\text {app }}$ are typically made near $y=1 / 2$ to have well-defined $y$ and $1-y$ values. Panel (c) shows the typical correction factors for measurements in the (immediate) vicinity of $y=1 / 2$, as a function of the parameter $\alpha$.

$\beta=e^{\theta}$ in the low and $\epsilon=e^{-\theta}$ in the high-temperature limit. With these substitutions, the coefficients in the master equation become polynomial, and limiting solutions for $y$ in the low temperature and $1-y$ in the high-temperature limit can be obtained by Taylor expansion around the point $(0,0)$. These are

$$
\begin{gathered}
y=\frac{1}{\alpha} e^{\theta}-\left(\frac{2+\alpha}{2 \alpha^{3}}\right) e^{2 \theta}+\cdots . \text { for } \theta \rightarrow-\infty, \\
y=1-\left[2 e^{-\theta}+(2 \alpha-8) e^{-2 \theta}+\cdots\right] \text { for } \theta \rightarrow \infty .
\end{gathered}
$$

The same result can be obtained using the differential transform method. Using Eqs. (16) and (23), it follows that

$$
\begin{aligned}
& \kappa_{y=0}=2, \\
& \kappa_{y=1}=1 .
\end{aligned}
$$

\section{E. Analytic solution in the $\alpha \rightarrow 0$ limit}

For very slow temperature increase $(\alpha \rightarrow 0)$ the master Eq. (24) becomes a simple quadratic equation in $y$ that can readily be solved:

$$
y=\frac{\sqrt{\beta^{2}+8 \beta}-\beta}{4}=\frac{\beta}{4}\left(\sqrt{1+\frac{8}{\beta}}-1\right), \quad \beta=e^{\theta} .
$$

For temperatures far below and above $T_{m}$, this can be approximated by the simpler expressions $y=\sqrt{\beta / 2}$ and $y=1-$ $2 / \beta$, respectively. Without approximation to Eq. (32), analytic expressions can be derived for the descriptors of Eq. (32) (for the details, see the Appendix):

$$
\begin{gathered}
\theta_{\mathrm{av}}=-1+\ln 2 \\
\theta_{\text {med }}=0 \\
\theta_{\text {peak }}=\ln \left(\frac{4}{1+\sqrt{2}}\right) \\
\Delta \theta=\sqrt{\frac{2 \pi^{2}}{3}+1} \\
\Delta \theta_{1 / 2}=\frac{1}{2} \ln \frac{[1-\sqrt{8}-\sqrt{3(\sqrt{32}-5)}]^{2}-(\sqrt{32}-6)^{2}}{\left[1-\sqrt{8}+\sqrt{3(\sqrt{32}-5)]^{2}}-(\sqrt{32}-6)^{2}\right.} \\
s=-\frac{12 \zeta(3)+2}{\left(\frac{2 \pi^{2}}{3}+1\right)^{3 / 2}} \\
\kappa=1 \text { for all } \theta \\
S_{\text {dis }} / R=0
\end{gathered}
$$

The lack of newly generated entropy is of course expected under quasiequilibrium conditions but can also be formally deduced from Eq. (22) together with the result for $\theta_{\mathrm{av}}$. Numerical values for the quantities in Eqs. (33)-(38) are given in Table I. The analytic results agree very well with the results from numerical integration of the master Eq. (24) in the $\alpha \rightarrow 0$ limit (Fig. 2). 
TABLE I. Parameters in this work and their values in the $\xi \rightarrow 0$ limit. All values in the table have been calculated using analytical formulas, or, in the case of $\theta_{1 / 2}$ in the large $\alpha$ limit, numerically from the analytical solution $y(\theta)$. The results agree very well with estimates from Figs. 2 and 3 , which depend on the numerical solution of the master equation.

\begin{tabular}{|c|c|c|c|c|c|c|}
\hline Quantity & Definition & Abbr & $\alpha \rightarrow 0$ & $\alpha=1$ & $\alpha=2$ & $\alpha \gg 2$ \\
\hline \multirow[t]{3}{*}{ Peak position } & Average or expectation value & $\theta_{\mathrm{av}}$ & -0.31 & 0.35 & 0.69 & $-0.58+\ln \alpha$ \\
\hline & Median & $\theta_{\text {med }}$ & 0.00 & 0.35 & 0.69 & $-0.37+\ln \alpha$ \\
\hline & Maximum & $\theta_{\text {peak }}$ & 0.50 & 0.35 & 0.69 & $\ln \alpha$ \\
\hline \multirow{2}{*}{ Peak width } & Standard deviation & $\Delta \theta$ & 2.75 & 2.02 & 1.81 & 1.28 \\
\hline & Half width at half maximum & $\Delta \theta_{1 / 2}$ & 2.55 & 2.21 & 1.76 & 1.23 \\
\hline Peak shape & Skewness & $s$ & -0.79 & 0 & 0 & -1.14 \\
\hline \multirow[t]{3}{*}{ Enthalpy correction } & $\Delta H_{\text {app }} /\left.\Delta H\right|_{y \rightarrow 0}$, low-temperature limit & $\kappa_{y=0}$ & 1.00 & 2.00 & 2.00 & 2.00 \\
\hline & $\Delta H_{\text {app }} /\left.\Delta H\right|_{y=1 / 2}$, typical value & $\kappa_{y=1 / 2}$ & 1.00 & 1.24 & 1.50 & 2.08 \\
\hline & $\Delta H_{\text {app }} /\left.\Delta H\right|_{y \rightarrow 1}$, high-temperature limit & $\kappa_{y=1}$ & 1.00 & 1.00 & 1.00 & 1.00 \\
\hline Dissipated entropy & Overall entropy increase & $S_{\mathrm{dis}} / R$ & 0.00 & 0.65 & 1.00 & $-0.27+\ln \alpha$ \\
\hline
\end{tabular}

\section{F. Analytic solution for $\alpha=1$}

The numerical calculations in Fig. 2 indicate that for $\alpha=1$, the skewness $s=0$, and $\theta_{\mathrm{av}}, \theta_{\text {med }}$, and $\theta_{\text {peak }}$ are all equal to $\ln \sqrt{2}$ to high precision. This suggests that for $\alpha=1$, $y\left(\delta_{1}+\ln \sqrt{2}\right)-1 / 2$ may be an odd function of $\delta_{1}$, and its derivative with respect to temperature an even function of $\delta_{1}$. With this assumption and the abbreviation $y=1 / 2+z$, and master Eq. (24), the equality of $d y / d \theta$ values on either side of the peak at equal distance can be expressed as

$$
\begin{aligned}
& -2\left(\frac{1}{2}+z\right)^{2}+\left(\frac{1}{2}-z\right) \sqrt{2} e^{\delta_{1}} \\
& =-2\left(\frac{1}{2}-z\right)^{2}+\left(\frac{1}{2}+z\right) \sqrt{2} e^{-\delta_{1}} .
\end{aligned}
$$

This can be rearranged to

$$
\begin{gathered}
y=\frac{1}{2}\left[1+\frac{\sinh (\theta-\ln \sqrt{2})}{\sqrt{2}+\cosh (\theta-\ln \sqrt{2})}\right]=\frac{e^{\theta}+2}{e^{\theta}+2 e^{-\theta}+4}, \\
\frac{d y}{d \theta}=\frac{1}{2} \frac{1+\sqrt{2} \cosh (\theta-\ln \sqrt{2})}{[\sqrt{2}+\cosh (\theta-\ln \sqrt{2})]^{2}}
\end{gathered}
$$

Tedious, but straightforward calculation confirms that a solution of master Eq. (24) has indeed been found. The characteristic quantities are

$$
\begin{gathered}
\theta_{\mathrm{av}}=\theta_{\text {med }}=\theta_{\text {peak }}=\ln \sqrt{2}, \\
\Delta \theta=\sqrt{\frac{[\ln (\sqrt{2}+1)]^{2}}{2}+\frac{[\ln (\sqrt{2}-1)]^{2}}{2}+\frac{\pi^{2}}{3},} \\
\Delta \theta_{1 / 2}=\operatorname{acosh}\left(2+2 \sqrt{1+\frac{1}{\sqrt{2}}}\right), \\
s=0, \\
\kappa_{y=1 / 2}=3(\sqrt{2}-1), \\
S_{\mathrm{dis}} / R=1-\ln \sqrt{2} .
\end{gathered}
$$

The result for $\Delta \theta$ was obtained using the maxima mathematics package. For the calculation of $\Delta \theta_{1 / 2}$, note that the maximal value of $d y / d \theta$ is $(\sqrt{2}-1) / 2$. Therefore, $\cosh \Delta \theta_{1 / 2}$ can be determined by solving a quadratic equation. For cal- culation of $S_{\text {dis }} / R$, Eq. (27) was used. A general expression for $\kappa$ at any $\theta$ can of course also be derived from Eq. (42), but the expression is quite tedious and therefore omitted here. Numerical values for the quantities in Eqs. (44)-(49) are given in Table I. The analytic results agree very well with the results from numerical integration of the master Eq. (24).

\section{G. Analytic solution for $\alpha=\mathbf{2}$}

The numerical calculations in Fig. 2 suggest that for $\alpha=2$, the skewness $s=0$, and $\theta_{\mathrm{av}}, \theta_{\mathrm{med}}$, and $\theta_{\text {peak }}$ are all equal to $\ln 2$. Proceeding analogously to the $\alpha=1$ case, one guesses that $y\left(\delta_{2}+\ln 2\right)-1 / 2$ is an odd function of $\delta_{2}$, and its derivative with respect to temperature an even function of $\delta_{2}$. One therefore has with the abbreviation $y=1 / 2+z$ :

$$
\begin{aligned}
- & 2\left(\frac{1}{2}+z\right)^{2}+2\left(\frac{1}{2}-z\right) e^{\delta_{2}} \\
& =-2\left(\frac{1}{2}-z\right)^{2}+2\left(\frac{1}{2}+z\right) e^{-\delta_{2}} .
\end{aligned}
$$

This can be rearranged to

$$
y=\frac{1}{2}\left(1+\frac{\sinh (\theta-\ln 2)}{1+\cosh (\theta-\ln 2)}\right)=\frac{e^{\theta}+1}{e^{\theta}+2 e^{-\theta}+3} .
$$

Taking into account the rules $\cosh \delta=-1+2 \cosh ^{2}(\delta / 2)$ and $\sinh \delta=2 \sinh (\delta / 2) \cosh (\delta / 2)$ for any $\delta$, the expression for $y$ can be further simplified to

$$
\begin{aligned}
& y=\frac{1}{2}\left[1+\tanh \left(\frac{\theta-\ln 2}{2}\right)\right]=\frac{1}{1+2 e^{-\theta}}, \\
& \frac{d y}{d \theta}=\frac{1}{4} \operatorname{sech}^{2}\left(\frac{\theta-\ln 2}{2}\right)=\frac{2 e^{-\theta}}{\left(1+2 e^{-\theta}\right)^{2}} .
\end{aligned}
$$

With these expressions, it is then easy to verify that $y(\theta)$ indeed satisfies the master Eq. (24), validating the assumption needed for the derivation of $y$. The characteristic quantities can then be straightforwardly calculated:

$$
\begin{gathered}
\theta_{\mathrm{av}}=\theta_{\mathrm{med}}=\theta_{\text {peak }}=\ln 2, \\
\Delta \theta=\pi / \sqrt{3}, \\
\Delta \theta_{1 / 2}=2 \ln (\sqrt{2}+1),
\end{gathered}
$$




$$
\begin{gathered}
s=0, \\
\kappa=1+\frac{2 e^{-\theta}}{1+2 e^{-\theta}}, \\
\kappa_{y=1 / 2}=3 / 2, \\
S_{\text {dis }} / R=1 .
\end{gathered}
$$

Note that the calculation of $\Delta \theta$ uses the well-known integrals $\int_{-\infty}^{\infty} \operatorname{sech}^{2} x d x=2$ and $\int_{-\infty}^{\infty} x^{2} \operatorname{sech}^{2} x d x=\pi /(2 \sqrt{3})$. For the calculation of $S_{\text {dis }} / R$, the expression for $\theta_{\text {av }}$ was used in combination with Eq. (27). Numerical values for the quantities in Eqs. (54)-(56) are given in Table I. They again agree very well with the results from integration of the master equation. Notice that $\kappa$ approaches 2 for large negative $\theta$ (or $y \rightarrow 0$ ), and 1 for large positive $\theta$ (or $y \rightarrow 0$ ), also in agreement with the numerical results (Fig. 2).

\section{H. Approximate analytic solution for $\alpha \gg 2$}

In the $\alpha \gg 2$ regime, the term $-2 y^{2}$ in the master Eq. (24) is negligible compared to $(1-y) e^{\theta}$, in mathematical terms because $y \ll 1$ below the dissociation temperature and because the term $e^{\theta}$ dominates above the dissociation temperature. In physical terms, the $-2 y^{2}$ term can be neglected, because there is no time for reassociation when temperature increase is very fast. Therefore, the master Eq. (24) simplifies to $-d[\ln (1-y)] / d \theta=1 /(1-y)(d y / d \theta)=(1 / \alpha) e^{\theta}=$ $e^{\theta-\ln \alpha}$. Taking into account the boundary condition $y \rightarrow 0$ as $\theta \rightarrow-\infty$, this can be integrated to

$$
y=1-e^{-e^{\theta-\ln \alpha}} .
$$

This result is remarkable. For large $\alpha, y$ depends upon $\theta$ according to the (cumulative) Gumbel minimal value distribution [1]. This explains the $\alpha$-independent shape of the dissociation curve in the large $\alpha$ limit, and the logarithmic increase of dissociation temperature with $\alpha$. For large $\alpha$, the agreement with the exact numerical solution is excellent (Fig. 1). Statistical parameters can be taken directly from Gumbel theory, or in case of $\kappa_{\text {med }}$, derived with reference to Eq. (19):

$$
\begin{gathered}
\theta_{\mathrm{av}}=-\gamma+\ln \alpha, \\
\theta_{\text {med }}=\ln (\ln 2)+\ln \alpha, \\
\theta_{\text {peak }}=\ln \alpha, \\
\Delta \theta=\pi / \sqrt{6}, \\
s=-12 \sqrt{6} \zeta(3) / \pi^{3}, \\
\kappa_{y=1 / 2}=3 \ln 2, \\
S_{\text {dis }} / R=1-\ln 2-\gamma+\ln \alpha .
\end{gathered}
$$

In these equations, $\gamma \approx 0.577$ is the Euler-Mascheroni constant and $\zeta$ is Riemann's $\zeta$ function. The value for argument 3 is Apery's constant $\zeta(3) \approx 1.202$. Note that $\ln (\ln 2) \approx$ -0.367 and that therefore $\theta_{\mathrm{av}}$ is slightly smaller than $\theta_{\text {med }}$. The negative skewness describes the heavier left than right tail of $d y / d \theta$ (Fig. 1). Numerical values for the quantities in Eqs. (62)-(68) are given in Table I. As in the other cases, the Gumbel results agree well with the findings obtained by solving the master Eq. (24) numerically. Large $\alpha$ estimates for peak position and width, and the van't Hoff correction factor $\kappa_{\text {med }}$ are fairly accurate for $\alpha>10$. The large $\alpha$ limit for dissociation curve skewness becomes relevant for $\alpha>100$. Notice that $\kappa_{y=1}$ cannot be calculated from Eq. (61), because the large $\theta$ limit has to be taken before the large $\alpha$ limit.

\section{Approximate analytic solution for all $\alpha$ and $\theta$}

A surprisingly useful approximate solution for all $\alpha$ and $\theta$ can be found by noticing a similarity in the expressions for the dissociation constant at $\alpha=0$ and $\alpha=2$. Define

$$
\tilde{K}=y^{2} /(1-y) \text {. }
$$

Hence, $\tilde{K}=K_{\text {app }} / c_{0}$ for heteroduplexes and $\tilde{K}=K_{\text {app }} /\left(4 c_{0}\right)$ for homoduplexes. For the special cases $\alpha=0$ and $\alpha=2, \tilde{K}$ can be readily calculated:

$$
\tilde{K}= \begin{cases}\frac{1}{2} e^{\theta} & \text { if } \alpha=0, \\ \frac{1}{2} e^{\theta} /\left(1+2 e^{\theta}\right) & \text { if } \alpha=2 .\end{cases}
$$

This suggests the following form for any $\alpha$ :

$$
\tilde{K}=\frac{1}{2}\left(\frac{e^{\theta}}{1+f(\alpha) e^{-\theta}}\right) .
$$

The function $f(\alpha)$ is yet to be determined, but from the special cases, it is already known that $f(0)=0$ and $f(2)=2$. Once $f$ is known, $\tilde{K}$ can be used to determine $y$ :

$$
y=\frac{-\tilde{K}+\sqrt{\tilde{K}^{2}+4 \tilde{K}}}{2} .
$$

Note that at $\theta_{\text {med }}$ and $\kappa_{y=1 / 2}$ can be calculated. $\tilde{K}=1 / 2$. Then $\theta_{\text {med }}$ can be determined by solving a quadratic equation:

$$
\begin{gathered}
\theta_{\text {med }}=\ln \left(\frac{1+\sqrt{1+4 f(\alpha)}}{2}\right), \\
\kappa_{y=1 / 2}=1+2 \alpha^{2}\left(\frac{1}{1+\sqrt{1+4 f(\alpha)}}\right)^{2} .
\end{gathered}
$$

To define $f(\alpha)$ better, one can look at the low-temperature expansion $y=e^{2 \theta} / \alpha^{2}+\cdots$ to conclude that

$$
f(\alpha)=\alpha^{2} / 2 \text {. }
$$

The solution $f(\alpha)=\alpha^{2} / 2$ is accurate for $\alpha=0, \alpha=2$, and a good approximation for large $\alpha$. However, it is not exact throughout. For $\alpha=1$, the formula predicts $\theta_{\text {med }}=$ $\ln (1+\sqrt{3})-\ln 2 \approx 0.31$, compared with the exact answer $\ln \sqrt{2} \approx 0.35$. It is possible to choose $f(\alpha)$ so that $\theta_{\text {med }}$ is exact for $\alpha=1$, at the cost of making the low-temperature asymptotic expansion inconsistent. The alternative $f(\alpha)$ is

$$
f(\alpha)=(\sqrt{2}-1) \alpha^{2}+(3-2 \sqrt{2}) \alpha .
$$

Both choices for $f(\alpha)$ lead to very similar large- $\alpha$ approximations for $\theta_{\text {med }}$, namely, $\theta_{\text {med }} \approx \ln \alpha-\ln \sqrt{2} \approx \ln \alpha-0.35$, when $f(\alpha)$ is chosen according to Eq. (75), and $\theta_{\text {med }} \approx \ln \alpha+$ $[\ln (\sqrt{2}-1)] / 2 \approx \ln \alpha-0.44$, when $f(\alpha)$ is chosen according to Eq. (76). For $\theta_{\text {med }}$, both guesses of $f(\alpha)$ lead to good 

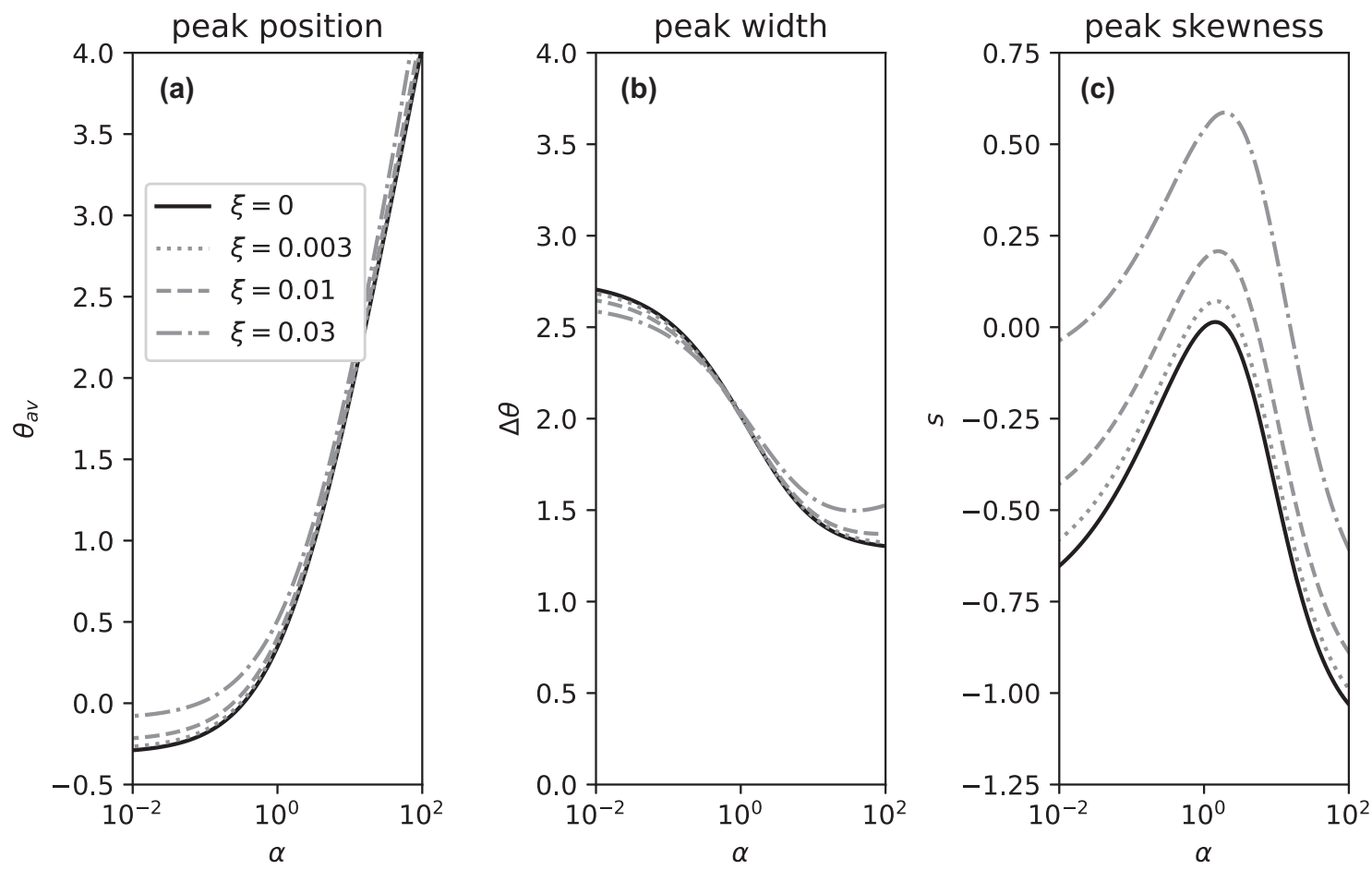

FIG. 4. Dissociation curve characteristics for small finite $\xi$. The legend in panel (a) applies to all panels.

approximations. For $\kappa_{y=1 / 2}$, both choices of $f$ reproduce the dependence on $\theta$ qualitatively, but lead to the wrong limiting value for large $\alpha$.

\section{CORRECTIONS FOR SMALL FINITE $\xi$}

In practice, the $\xi \rightarrow 0$ limit is of course always an approximation. In the following, corrections for small finite $\xi$ are considered. We require that the transition is well defined as before, and additionally, that the $\xi$ expansion for $\Theta$ in terms of $\theta$ (or of $\theta$ in terms of $\Theta$ ) is meaningful. Hence,

$$
\begin{gathered}
\xi \ll 1, \\
|\ln \alpha| \ll 1 / \xi .
\end{gathered}
$$

\section{A. Dissociation curve}

Dissociation curves remain roughly similar for small finite $\xi$. Differences are better seen with respect to characteristic quantities describing the dissociation curves. To account for $y_{\max }<1$, "statistical" descriptors $\theta_{\mathrm{av}}, \Delta \theta$, and $s$ were calculated with reference to $d\left(y / y_{\max }\right) / d \theta$, rather than $d y / d \theta$, as the "probability density" (Fig. 4). The $\theta_{\text {av }}$ value increases slightly with $\xi$ for fixed $\alpha$, but differences are relatively small [Fig. 4(a)]. The dependence of $\Delta \theta$ on $\xi$ for fixed $\alpha$ depends on the regime: For small $\alpha$, there is a slight decrease, for large $\alpha$, a small increase is calculated [Fig. 4(b)]. More substantial changes occur in curve shape. While dissociation curves are always left-skewed in the $\xi \rightarrow 0$ limit, they can be rightskewed for finite $\xi$ [Fig. 4(c)].

\section{B. Limiting values for van't Hoff $\kappa$}

Like dissociation curves, van't Hoff plots for $\xi>0$ are roughly similar to those for $\xi \rightarrow 0$. In the high-temperature regime, the usual limit for $\kappa$ applies:

$$
\kappa=1 \text { for high temperature. }
$$

In the low-temperature regime, $\kappa$ reaches a limiting value that is larger than in the $\xi \rightarrow 0$ limit. To understand this effect, it is necessary to obtain an approximate expression for $y$ in the low-temperature (small $y$ ) regime. As $d / d \theta=$ $(1-\xi \Theta)^{2} d / d \Theta$ according to Eq. (7), $y \approx C e^{\Theta} /(1-\xi \Theta)^{2}$ with unknown constant $C$ is a reasonable low-temperature approximation. Combining this result with Eqs. (16) and (18), and taking into account that the change in $K_{\text {app }}$ is dominated by changes of the numerator in Eq. (16), one can derive that $\kappa=d[2 \Theta-4 \ln (1-\Theta \xi)] / d \Theta$. As $1-\xi \Theta=1 /(1+$ $\xi \theta$ ) according to Eq. (7), it follows that

$$
\kappa=2+4 \xi(1+\xi \theta) \text { for low temperature. }
$$

In moderately low temperature $|\theta \xi| \ll 1$, and therefore $\kappa \approx 2+4 \xi$, in good agreement with the numerical result [Fig. 5(a)].

\section{Dissipated entropy}

Surprisingly, the newly generated entropy $S_{\text {dis }}$, calculated according to Eq. (22), depends barely if at all on $\xi$ for fixed $\alpha$ [Fig. 5(c)]. The independence of $S_{\text {dis }}$ from $\xi$ is trivial in the quasistationary $\alpha \rightarrow 0$ limit, since new entropy is not generated in this regime, for any $\xi$. The near or complete independence of $S_{\mathrm{dis}}$ from $\xi$ is remarkable in the large $\alpha$ regime. No exact reason was found, but a tentative explanation can be given. According to Eq. (22),

$$
\frac{1}{R} \frac{d S_{\mathrm{dis}}}{d \xi}=\frac{d}{d \xi} \int_{-1 / \xi}^{\infty} \frac{\theta}{1+\xi \theta} \frac{d y}{d \theta} d \theta=\frac{-\left\langle\theta^{2}\right\rangle+\frac{d}{d \xi}\langle\theta\rangle}{y_{\min }} .
$$



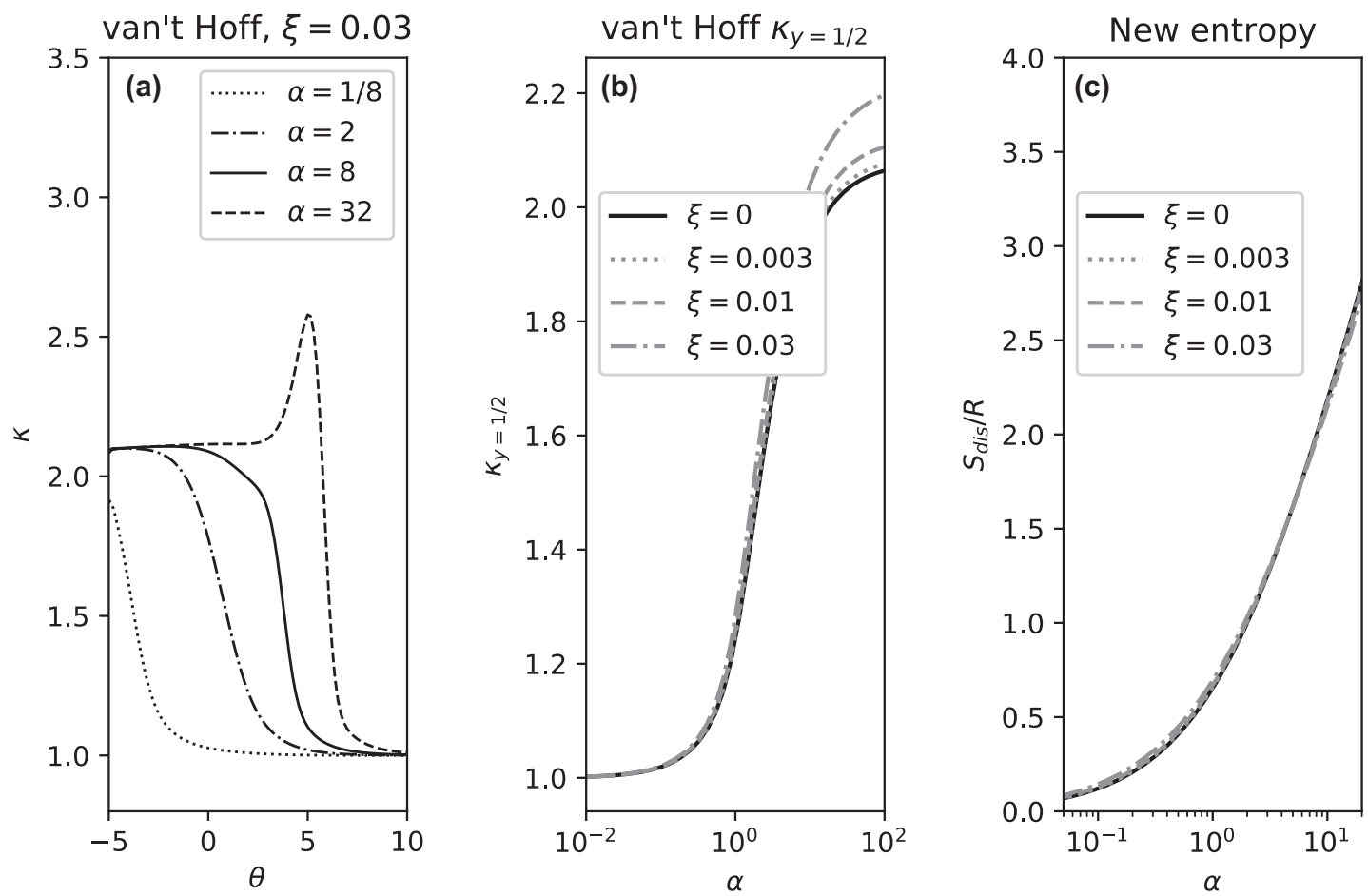

FIG. 5. Thermodynamic values for small finite $\xi$. The legend in panel (a) applies to all panels.

The integral boundary-dependent term of the derivative has been neglected, because $d y / d \theta$ approaches 0 faster than $\theta /(1+\xi \theta)$ approaches $\infty$. Using variable substitution, it can be shown that $(\ln \alpha)^{2}$ is the leading-order term in $\alpha$ for both $\left\langle\theta^{2}\right\rangle$ and $d\langle\theta\rangle / d \xi$. Therefore, $S_{\text {dis }} / R$ is less $\xi$-dependent than $\theta_{\mathrm{av}}=\langle\theta\rangle$ for large $\alpha$, in agreement with the numerical results [compare Fig. 5(c) with Fig. 4(a)].

\section{APPLICATION TO DNA MELTING}

The melting of short DNA duplexes has been very successfully described by a two-state model, at least in quasiequilibrium conditions [2]. The rate constant $k_{\text {as }}$ for strand association is only weakly temperature-dependent [3]. It increases with temperature up to roughly the melting temperature, and then decreases again [4]. The rate of double strand dissociation $k_{\text {dis }}$ follows an Arrhenius law [11]. The model described in this work idealizes this behavior. Based on an average melting entropy of $23 \mathrm{cal} /(\mathrm{molK})$, and the value for the Boltzmann constant $R$ of $2 \mathrm{cal} /(\mathrm{molK})$, one can estimate $\xi=$ $2 /(23 n) \approx 0.09 / n$, where $n$ is the number of base pairs $(\mathrm{bp})$ in the duplex. The parameter $\xi<0.03$ for $n>3$, and hence $\xi \rightarrow$ 0 result apply to a very good approximation. The longer DNA duplexes, the smaller $\xi$. However, for very long duplexes, melting is not well approximated by a two-step reaction, and better described by more complicated models [5-9].

\section{A. Changes in melting rate}

Changes in melting (heating) rate for fixed salt concentration translate to changes in $\alpha$ according to Eq. (9). In the large $\alpha$ regime, Eq. (62) predicts a logarithmic increase of $T_{\text {melt }}$ with $\Gamma$ and hence $\alpha$. The expected magnitude of change is

$$
\frac{d T_{\mathrm{melt}}}{d(\ln \Gamma)}=\frac{\Delta T_{\mathrm{vH}}}{4}=\frac{R T_{m}^{2}}{\Delta H}=\frac{R T_{m}^{2}}{\Delta H_{\mathrm{bp}}} \frac{1}{n} .
$$

In this equation, $n$ is the number of base pairs, and $\Delta H_{\mathrm{bp}}$ the average melting enthalpy per base pair. As $T_{m}$ depends only weakly on DNA length for duplexes in this length range, $d T_{\text {melt }} / d \ln \Gamma$ should be approximately inversely proportional to DNA length. The dependence of melting temperature on the rate of temperature increase $\Gamma$ for fixed salt concentration $a$ has recently been measured for three different DNA duplexes of length 51, 100, and $272 \mathrm{bp}$ [10]. A logarithmic dependence of $T_{\text {melt }}$ on $\Gamma$, and a decrease of $d T_{\text {melt }} / d \ln \Gamma$ with $n$ are observed, as predicted for the large $\alpha$ regime. However, the dependence of $d T_{\text {melt }} / d \ln \Gamma$ on $n$ is weaker than expected, presumably because the assumption of a single-step dissociation reaction breaks down for the longer duplexes [Fig. 6(a)].

\section{B. Changes in ionic strength}

Complementary DNA strands associate more rapidly in high than low salt, due to screening of backbone repulsion in high, but not low salt. Quantitatively, the dependence can be described by a power law, with a DNA-length-dependent exponent $p$ with values typically between 2 and $4[11,12]$. At $T_{m}, k=2 k_{\mathrm{as}} c_{0}$ for homoduplexes, and $k=k_{\mathrm{as}} c_{0} / 2$ for heteroduplexes as derived in Sec. I B. For fixed heating rate $\Gamma$ and varying salt concentration $a, \alpha$ can therefore be expressed according to Eq. (9) as

$$
\alpha=\left(\frac{a}{a_{0}}\right)^{-p},
$$

where $a_{0}$ is a suitably chosen constant (with units of salt concentration). As $p$ is positive, $\alpha$ is small in high salt and large in low salt. The half-width of the melting transition can then be expressed in terms of the dimensionless $\theta_{1 / 2}(\alpha)$ as

$$
\Delta T_{1 / 2}=\frac{\Delta T_{\mathrm{vH}}}{4} \Delta \theta_{1 / 2}\left[\left(\frac{a}{a_{0}}\right)^{-p}\right] .
$$



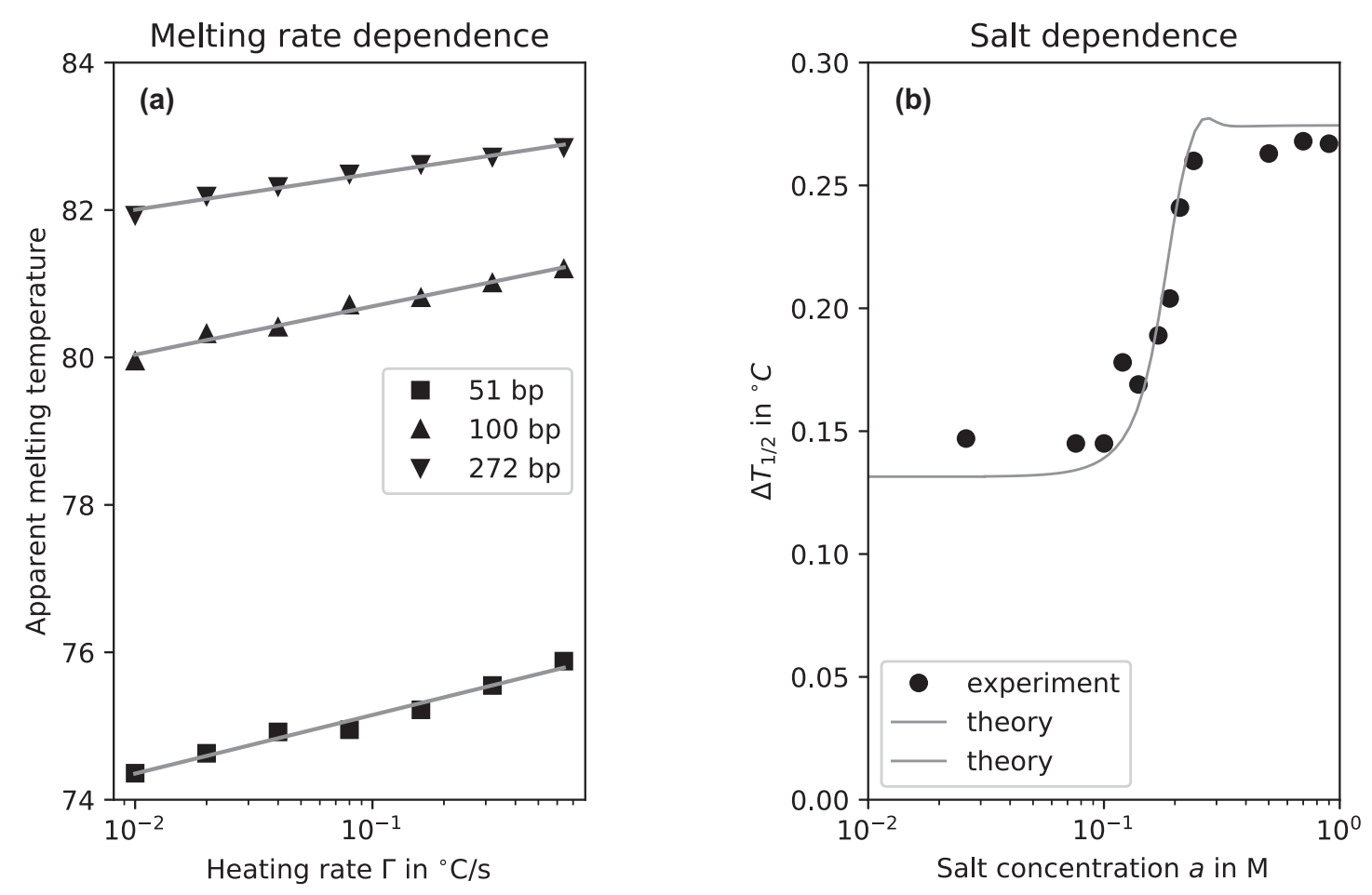

FIG. 6. Change of apparent melting temperature (a) and melting width (b) with changing $\alpha$. In the experiment shown in (a), carried out by Li and colleagues [10], three DNA duplexes of different length were studied, and $\alpha$ was varied by changing the melting rate $\Gamma$ for fixed salt concentration $a$. Gray lines represent best least square fits assuming a logarithmic dependence of apparent melting temperature on $\alpha$ or $\Gamma$, as predicted by Eq. (82). In the experiment shown in (b), carried out by Hillen and colleagues [13], $\alpha$ was varied by changing the salt concentration $a$ for fixed melting rate $\Gamma$. Data are shown for a 300-mer duplex. The gray curve illustrates the theoretical expectation based on Eq. (84) for fit parameters $p=3.7, a_{0}=0.2 M$ and $\Delta T_{\mathrm{vH}}=0.43^{\circ} \mathrm{C}$. The $p$ value is typical for a DNA of this length [12], and the $\Delta T_{\mathrm{vH}}$ value is in fair agreement with the expected $\Delta T_{\mathrm{vH}}=0.34^{\circ} \mathrm{C}$ based on the melting point of the 300-mer duplex assuming a melting entropy per base pair of $23 \mathrm{cal} /(\mathrm{molK})$. Hillen and colleagues also studied two shorter duplexes that exhibit qualitatively similar behavior. Data for these are not shown, because the change in melting width occurs at the edge of the studied range of salt concentrations.

In light of the results for $\theta_{1 / 2}(\alpha)$ in Figs. 2(b) and 4(b), the equation predicts an approximately constant melting halfwidth $\Delta T_{1 / 2}$ in the (highly irreversible) low salt regime, and an approximately twice larger $\Delta T_{1 / 2}$ in the (quasiequilibrium) high salt regime. This behavior is indeed observed experimentally [13]. However, the change $\Delta T_{1 / 2}$ from low to high salt is slightly smaller than calculated, due to the neglected increase of $\Delta H$ (and concomitant decrease of $\Delta T_{\mathrm{vH}}$ and thus $\Delta T_{1 / 2}$ ) with growing salt concentration [14] [Fig. 6(b)].

\section{DISCUSSION}

\section{A. Master equations and interpretation of their parameters}

In this work, temperature-increase-driven dissociation of homo- or heteroduplexes is considered. It is assumed that dissociation is controlled by an Arrhenius law and counteracted by a temperature-independent association reaction. The model leads to the master differential Eq. (8), which is dependent on two parameters, $\xi$ and $\alpha$. The dimensionless parameter $\xi$ is a material parameter that measures the abruptness of the dissociation reaction, under equilibrium conditions, and is the smaller the more abruptly the transition occurs. According to Eq. (21) the inverse of $\xi$ equals the dissociation entropy under equilibrium conditions, $\Delta S_{0}$, except for a small constant offset. The dimensionless parameter $\alpha$ describes the rate of temperature increase, in units of temperature and time set by the properties of the system. For all $\xi$, the newly generated entropy $S_{\text {dis }}$ increases with $\ln \alpha$. In the $\xi \rightarrow 0$ limit, and for large $\alpha$, the relationship is linear according to Eq. (68). In this regime, $\ln \alpha$ equals the newly generated entropy in units of the Boltzmann constant, $S_{\text {dis }} / R$, except for a constant offset. Numerical calculations show that $\ln \alpha$ remains a good measure for $S_{\text {dis }} / R$ in the large $\alpha$ limit also for finite $\xi$ (Fig. 5). Therefore, $\alpha$ can be regarded as an "irreversibility" parameter.

\section{B. Special solutions in the $\xi \rightarrow 0$ limit}

In this work, the focus is on fairly abrupt transitions $(\xi<$ 0.03 ) that are well described by the $\xi \rightarrow 0$ limit (Figs. 4 and 5). In this limit, the two-parameter master Eq. (8) becomes the one-parameter master Eq. (24), which has the form of a Ricatti equation. Such equations cannot be solved analytically in general, but solutions can sometimes be found in special cases. Here, solutions were identified for the simpler Eq. (24) for $\alpha \rightarrow 0, \alpha=1, \alpha=2$ and $\alpha \gg 2$. For $\alpha \rightarrow 0$ and $\alpha \gg$ 2 , analytic solutions are possible because one of the terms in master Eq. (24) can be neglected. For $\alpha=1$ and $\alpha=2$, numerical calculations indicate that $d y(\theta) / d \theta$ is not skewed. The absence of skewness is necessary but not sufficient for a 
symmetric solution (i.e., a solution satisfying $\left[y\left(\theta_{\text {med }}+\delta\right)-\right.$ $1 / 2]=-\left[y\left(\theta_{\text {med }}-\delta\right)-1 / 2\right]$ for all $\delta$ with suitably chosen $\left.\theta_{\text {med }}\right)$. Assuming the symmetry, an analytical expression for $y(\theta)$ can be derived, and then justified retrospectively by the demonstration that the solution [Eq. (42) for $\alpha=1$ and (76) for $\alpha=2$ ] happens to satisfy the differential Eq. (24). Based on the closed expressions for $\tilde{K}=y^{2} /(1-y)$ for $\alpha \rightarrow 0$ and $\alpha=2$, approximate expressions for $\tilde{K}$, given in Eq. (71), and for $y(\theta)$, given in Eq. (72), can be guessed. With $f(\alpha)=$ $\alpha^{2} / 2$, the expressions are exact for $\alpha=0$ and $\alpha=2$, and approximate for other $\alpha$ values. The approximate solution for $y(\theta)$ predicts surprisingly well peak position [Fig. 2(a)], peak width [Fig. 2(b)], and even skewness [Fig. 2(c)]. It also leads to a closed form estimate of $\kappa_{y=1 / 2}$ that qualitatively reflects the behavior of the system, even though the large $\alpha$ limiting value is underestimated [Fig. 3(c)]. Attempts to identify continuous (Lie group) symmetries of master Eq. (24) to derive an exact general solution for arbitrary $\alpha$ were not successful.

\section{A universal statistical distribution in the $\xi \rightarrow 0$ and $\alpha \gg 2$ limit}

Equation (61) in this work shows that $y(\theta)$ in the $\xi \rightarrow 0$ and $\alpha \gg 2$ limit has the form of a Gumbel distribution [1]. Gumbel distributions, along with Fréchet and Weibull distributions, are the universal limiting distributions for extremes [15]. Mathematically, $y(\theta)$ has the form of a Gumbel distribution, because the association term in the master Eq. (24) can be neglected. As the same argument can be made for analogous $n$-component systems, the result should generalize to such systems as well. From a physical point of view, the finding is remarkable, because there is no obvious process of extreme value selection in the studied simple model.

\section{Dissociation temperature and width in the highly irreversible $(\alpha \gg 2)$ regime}

This work demonstrates that the the dissociation temperature (for any of the measures $\theta_{\mathrm{av}}, \theta_{\text {med }}$, or $\theta_{\text {peak }}$ ) increases with the logarithm of $\alpha$ for $\alpha \gg 2$ [Figs. 2(a) and 4(a)]. This logarithmic dependence can be explained qualitatively, assuming only that the dissociation reaction occurs in a temperature interval corresponding to the van't Hoff width $\Delta T_{\mathrm{vH}}$. The time $\Delta t=\Delta T_{\mathrm{vH}} / \Gamma$ to traverse this temperature interval must suffice for the homo- or heteroduplex to dissociate. Therefore, $\Delta t k e^{\theta_{\text {dis }}}=\Delta T_{\mathrm{vH}} k e^{\theta_{\text {dis }}} / \Gamma \approx 1$ or $\theta_{\text {dis }}=\ln \left[\Gamma /\left(k \Delta T_{\mathrm{vH}}\right)\right]$, which reproduces the exact result Eq. (62) except for the small constant offset. For the special case of DNA melting, a logarithmic dependence of melting temperature on melting rate has previously been predicted in the context of a model of DNA melting that involves separate steps of bubble formation and growth [16].

This work also shows that the temperature range in which most of the dissociation reaction occurs is twice narrower in the highly irreversible (large $\alpha$ ) than in the quasiequilibrium (small $\alpha$ ) regime [Figs. 2(b) and 4(b)]. Qualitatively, this happens because re-association plays a role in the quasiequilibrium, but not the highly irreversible regime. For the example of DNA, the transition from the quasiequilibrium to the highly irreversible regime can be made for fixed heating rate by varying the salt concentration, and with it the parameter $k$. In high salt, the repulsion between phosphodiester backbones is reduced, and association and dissociation rates are high, so that a fixed heating rate $\Gamma$ translates to a small $\alpha$. In low salt, association and dissociation are slow, and a fixed heating rate translates into a large $\alpha$. In the latter regime, the width of the melting region is twice smaller than in the former. This explains, for the first time to the author's knowledge, the salt-dependence of the melting width of DNA.

\section{E. van't Hoff versus calorimetric enthalpies}

It has been frequently noticed that van't Hoff and calorimetric enthalpies disagree $[17,18]$. The discrepancy has been traced to heat capacity effects [14], volume change effects [19], technical complications in calorimetry related to blank subtraction [20], and problems arising from propagation of experimental error [18]. Using the example of DNA melting, this work shows that departure from ideal quasiequilibrium conditions can also be a source of systematic discrepancies, which can exceed a factor of 2 [Figs. 3(b), 3(c), and 5(b)]. According to this work, nonequilibrium effects inflate the apparent van't Hoff enthalpy. In experiments, the opposite is typically seen. Therefore, the current analysis does not resolve the discrepancies, but it identifies an additional potential problem that can complicate the analysis.

\section{METHODS}

Numerical results were generated in python using the SciPy and NumPy packages. The ordinary differential Eq. (24) was solved using the differential equation solver odeint. Calculations are available as a Jupyter Noteboook in Google colab [21] and as Supplemental Material as a ipynb file [22]. Selected results were also independently checked using the GNU octave differential equation solver lsode or the Maxima fourth-order Runge-Kutta differential equation solver rk. The analytic results presented in the Appendix were first obtained using symbolic calculus as implemented in the Maxima computer algebra package. Experimental results are from Refs. [10,13].

\section{ACKNOWLEDGMENTS}

Helpful discussions on the practicalities of DNA melting in high-resolution melting analysis with M.Sc. Anna Fedenko, Dr. Malgorzata Perycz, Dr. Joanna Krwawicz, Dr. Malgorzata Wiweger, and Dr. Katarzyna Misztal are gratefully acknowledged. Work in the Bochtler laboratory was supported by the Polish National Science Centre (NCN, Grants No. 2011/02/A/NZ1/00052, No. 2014/13/B/NZ1/03991, No. 2014/14/M/NZ5/00558, and No. 2017/27/L/NZ2/03234), the Foundation for Polish Science (FNP, Grant No. POIR.04.04.00-00-5D81/17-00), and by the Polish National Agency For Academic Exchange (NAWA) Grant No. PPI/APM/2018/1/00034 within the framework of the International Academic Partnerships program. 


\section{APPENDIX}

\section{Calculations for the $\alpha \rightarrow 0$ limit}

The quantities $\theta_{\mathrm{av}}, \Delta \theta$, and $s$ can be expressed in terms of the first three moments of the variable of $\hat{\theta}$. We therefore need to calculate for $m=1,2,3$ :

$$
\left\langle\hat{\theta}^{m}\right\rangle=\int_{-\infty}^{\infty} \theta^{m} \frac{d y}{d \theta} d \theta=\int_{0}^{1} \theta^{m} d y .
$$

In the limit $\alpha \rightarrow 0$, the master Eq. (24) requires

$$
\theta=\ln 2+2 \ln y-\ln (1-y) .
$$

In this limit, $\left\langle\hat{\theta}^{m}\right\rangle$ can therefore also be expressed as

$$
\left\langle\hat{\theta}^{m}\right\rangle=\int_{0}^{1}[\ln 2+2 \ln y-\ln (1-y)]^{m} d y .
$$

The integrals encountered in its evaluation are known, and can be expressed, in some cases with the help of special values of Riemann's $\zeta$ function:

$$
\begin{gathered}
\int_{0}^{1}(\ln y)^{v} d y=\int_{0}^{1}[\ln (1-y)]^{v} d y= \begin{cases}-1 & \text { if } v=1, \\
2 & \text { if } v=2, \\
-6 & \text { if } v=3,\end{cases} \\
\int_{0}^{1} \ln y \ln (1-y) d y=2-\zeta(2)=2-\pi^{2} / 6, \\
\int_{0}^{1}(\ln y)^{2} \ln (1-y) d y=\frac{6 \zeta(3)+\pi^{2}+18}{3}, \\
\int_{0}^{1} \ln y[\ln (1-y)]^{2} d y=\frac{6 \zeta(3)+\pi^{2}+18}{3} .
\end{gathered}
$$

With these expressions, one readily calculates

$$
\begin{gathered}
\langle\hat{\theta}\rangle=-1+\ln 2, \\
\left\langle\hat{\theta}^{2}\right\rangle=(\ln 2)^{2}-2 \ln 2+2+2 \pi^{2} / 3,
\end{gathered}
$$

$$
\begin{aligned}
\left\langle\hat{\theta}^{3}\right\rangle= & -12 \zeta(3)+(\ln 2)^{3}-3(\ln 2)^{2} \\
& +\left(2 \pi^{2}+6\right) \ln 2-2 \pi^{2}-6 .
\end{aligned}
$$

Analytical expressions are also possible for higher moments, but the expressions become very unwieldy. Numerical values are

$$
\begin{gathered}
\langle\hat{\theta}\rangle \approx-0.307, \\
\left\langle\hat{\theta^{2}}\right\rangle \approx 7.674, \\
\left\langle\hat{\theta^{3}}\right\rangle \approx-23.431, \\
\left\langle\hat{\theta^{4}}\right\rangle \approx 306.711 .
\end{gathered}
$$

Based on the moments, one can calculate

$$
\begin{gathered}
\theta_{\mathrm{av}}=\langle\hat{\theta}\rangle=-1+\ln 2, \\
\Delta \theta=\sqrt{\left\langle\hat{\theta}^{2}\right\rangle-\langle\hat{\theta}\rangle^{2}}=\sqrt{\frac{2 \pi^{2}}{3}+1,} \\
s=\frac{\left\langle\hat{\theta}^{3}\right\rangle-3(\Delta \theta)^{2}\langle\hat{\theta}\rangle-\langle\hat{\theta}\rangle^{3}}{(\Delta \theta)^{3 / 2}}=-\frac{12 \zeta(3)+2}{\left(\frac{2 \pi^{2}}{3}+1\right)^{3 / 2}} .
\end{gathered}
$$

To determine the $\theta_{\text {peak }}$, it is useful to set $\psi=\sqrt{8 e^{-\theta}+1}$. It then follows that $d y / d \theta=2 /(\psi+1)-1 / \psi$. This is maximal for $\psi=1+\sqrt{2}$, which implies

$$
\theta_{\text {peak }}=\ln \left(\frac{4}{1+\sqrt{2}}\right),\left.\quad \frac{d y}{d \theta}\right|_{\text {peak }}=3-2 \sqrt{2} .
$$

Hence, $d y / d \theta=1 / 2 \cdot d y /\left.d \theta\right|_{\text {peak }}$ requires

$$
\psi=\frac{1-\sqrt{8} \pm \sqrt{3(\sqrt{32}-5)}}{\sqrt{32}-6}
$$

The expression for $\Delta \theta_{1 / 2}$ can then be directly deduced using the definition of $\psi$.
[1] E. J. Gumbel, Statistics of Extremes (Columbia University Press, New York, 1958).

[2] J. SantaLucia, Jr., A unified view of polymer, dumbbell, and oligonucleotide DNA nearest neighbor thermodynamics, Proc. Natl. Acad. Sci. USA 95, 1460 (1998).

[3] J. G. Wetmur and N. Davidson, Kinetics of renaturation of DNA, J. Mol. Biol. 31, 349 (1968).

[4] G. He, J. Li, H. Ci, C. Qi, and X. Guo, Direct measurement of single-molecule DNA hybridization dynamics with single-base resolution, Angew. Chem. Int. Ed. Engl. 55, 9036 (2016).

[5] A. Y. Grosberg and A. R. Khokhlov, Statistical Physics of Macromolecules (American Institute of Physics, Philadelphia, PA, 1994).

[6] B. H. Zimm, Theory of "melting" of the helical form in double chains of the DNA type, J. Chem. Phys. 33, 1349 (1960).

[7] D. Poland and H. A. Scheraga, Occurrence of a phase transition in nucleic acid models, J. Chem. Phys. 45, 1464 (1966).
[8] S. Ares, N. K. Voulgarakis, K. Ø. Rasmussen, and A. R. Bishop, Bubble Nucleation and Cooperativity in DNA Melting, Phys. Rev. Lett. 94, 035504 (2005).

[9] N. Theodorakopoulos, T. Dauxois, and M. Peyrard, Order of the Phase Transition in Models of DNA Thermal Denaturation, Phys. Rev. Lett. 85, 6 (2000).

[10] M. Li, R. A. Palais, L. Zhou, and C. T. Wittwer, Quantifying variant differences in DNA melting curves: Effects of length, melting rate, and curve overlay, Anal. Biochem. 539, 90 (2017).

[11] S. Ikuta, K. Takagi, R. B. Wallace, and K. Itakura, Dissociation kinetics of 19 base paired oligonucleotide-DNA duplexes containing different single mismatched base pairs, Nucl. Acids Res. 15, 797 (1987).

[12] J. L. Sikorav, H. Orland, and A. Braslau, Mechanism of thermal renaturation and hybridization of nucleic acids: Kramers' process and universality in Watson-Crick base pairing, J. Phys. Chem. B 113, 3715 (2009). 
[13] W. Hillen, T. C. Goodman, and R. D. Wells, Salt dependence and thermodynamic interpretation of the thermal-denaturation of small DNA restriction fragments, Nucl. Acids Res. 9, 415 (1981).

[14] I. Rouzina and V. A. Bloomfield, Heat capacity effects on the melting of DNA. 1. General aspects, Biophys. J. 77, 3242 (1999).

[15] S. Kotz and S. Nadarajah, Extreme Value Distributions: Theory and Applications (Imperial College Press, Distributed by World Scientific, London River Edge, NJ, 2000).

[16] V. V. Anshelevich, A. V. Vologodskii, A. V. Lukashin, and M. D. Frankkamenetskii, Slow relaxational processes in the melting of linear bio-polymers-A theory and its application to nucleicacids, Biopolymers 23, 39 (1984).

[17] J. W. H. Sutherland, Disagreement between calorimetric and van't Hoff enthalpies of assembly of protein supramolecular structures, Proc. Nat. Acad. Sci. USA 74, 2002 (1977).
[18] J. Tellinghuisen, Van't hoff analysis of $k$ degrees $(t)$ : How good...or bad? Biophys. Chem. 120, 114 (2006).

[19] I. A. Stepanov, The heats of chemical reactions: The van'tHoff equation and calorimetry, Z. Phys. Chem. 219, 1089 (2005).

[20] L. S Mizoue and J. Tellinghuisen, Calorimetric vs van't hoff binding enthalpies from isothermal titration calorimetry: $\mathrm{Ba}^{2+}$-crown ether complexation, Biophys. Chem. 110, 15 (2004).

[21] https://colab.research.google.com/drive/1p1s0nHDhPTZQ1Fz KjCAFJsRX0BnonFZV.

[22] See Supplemental Material at http://link.aps.org/supplemental/ 10.1103/PhysRevE.101.032405 for all numerical calculations in this work. The supplement is a Jupyter notebook file that can be viewed in Jupyter notebook or Jupyter laboratory. 